\title{
Sparse complete sets for coNP: Solution of the P versus NP problem
}

\author{
Frank Vega $\square$ 尽 (i) \\ CopSonic, 1471 Route de Saint-Nauphary 82000 Montauban, France
}

\begin{abstract}
$\mathrm{P}$ versus NP is considered as one of the most important open problems in computer science. This consists in knowing the answer of the following question: Is P equal to NP? A precise statement of the $\mathrm{P}$ versus NP problem was introduced independently by Stephen Cook and Leonid Levin. Since that date, all efforts to find a proof for this problem have failed. Another major complexity class is coNP. Whether NP $=$ coNP is another fundamental question that it is as important as it is unresolved. In 1979, Fortune showed that if any sparse language is coNP-complete, then $\mathrm{P}=\mathrm{NP}$. We prove there is a possible sparse language in coNP-complete. In this way, we demonstrate the complexity class $\mathrm{P}$ is equal to NP.
\end{abstract}

2012 ACM Subject Classification Theory of computation Complexity classes; Theory of computation Problems, reductions and completeness

Keywords and phrases Complexity Classes, Complement Language, Sparse, Completeness, Polynomial Time

\section{Introduction}

The $P$ versus $N P$ problem is a major unsolved problem in computer science [6]. This is considered by many to be the most important open problem in the field [6]. It is one of the seven Millennium Prize Problems selected by the Clay Mathematics Institute to carry a US $\$ 1,000,000$ prize for the first correct solution [6]. It was essentially mentioned in 1955 from a letter written by John Nash to the United States National Security Agency [1]. However, the precise statement of the $P=N P$ problem was introduced in 1971 by Stephen Cook in a seminal paper [6].

In 1936, Turing developed his theoretical computational model [19]. The deterministic and nondeterministic Turing machines have become in two of the most important definitions related to this theoretical model for computation [19]. A deterministic Turing machine has only one next action for each step defined in its program or transition function [19]. A nondeterministic Turing machine could contain more than one action defined for each step of its program, where this one is no longer a function, but a relation [19].

Another relevant advance in the last century has been the definition of a complexity class. A language over an alphabet is any set of strings made up of symbols from that alphabet [7]. A complexity class is a set of problems, which are represented as a language, grouped by measures such as the running time, memory, etc [7].

In the computational complexity theory, the class $P$ contains those languages that can be decided in polynomial time by a deterministic Turing machine [13]. The class $N P$ consists in those languages that can be decided in polynomial time by a nondeterministic Turing machine [13]. The biggest open question in theoretical computer science concerns the relationship between these classes: Is $P$ equal to $N P$ ? In 2012, a poll of 151 researchers showed that 126 $(83 \%)$ believed the answer to be no, $12(9 \%)$ believed the answer is yes, $5(3 \%)$ believed the question may be independent of the currently accepted axioms and therefore impossible to prove or disprove, $8(5 \%)$ said either do not know or do not care or don't want the answer to be yes nor the problem to be resolved [12]. 
It is fully expected that $P \neq N P$ [18]. Indeed, if $P=N P$ then there are stunning practical consequences [18]. For that reason, $P=N P$ is considered as a very unlikely event [18]. Certainly, $P$ versus $N P$ is one of the greatest open problems in science and a correct solution for this incognita would have a great impact not only for computer science, but for many other fields as well [1]. Whether $P=N P$ or not is still a controversial and unsolved problem [1]. In this work, we state some evidences which claim that the complexity class $P$ could be equal to $N P$.

\section{Basic Definitions}

Let $\Sigma$ be a finite alphabet with at least two elements, and let $\Sigma^{*}$ be the set of finite strings over $\Sigma$ [2]. A Turing machine $M$ has an associated input alphabet $\Sigma$ [2]. For each string $w$ in $\Sigma^{*}$ there is a computation associated with $M$ on input $w$ [2]. We say that $M$ accepts $w$ if this computation terminates in the accepting state, that is $M(w)=$ "yes" [2]. Note that $M$ fails to accept $w$ either if this computation ends in the rejecting state, that is $M(w)=$ "no", or if the computation fails to terminate [2].

The language accepted by a Turing machine $M$, denoted $L(M)$, has an associated alphabet $\Sigma$ and is defined by

$$
L(M)=\left\{w \in \Sigma^{*}: M(w)=\text { "yes" }\right\} .
$$

We denote by $t_{M}(w)$ the number of steps in the computation of $M$ on input $w$ [2]. For $n \in \mathbb{N}$ we denote by $T_{M}(n)$ the worst case run time of $M$; that is

$$
T_{M}(n)=\max \left\{t_{M}(w): w \in \Sigma^{n}\right\}
$$

where $\Sigma^{n}$ is the set of all strings over $\Sigma$ of length $n$ [2]. We say that $M$ runs in polynomial time if there is a constant $k$ such that for all $n, T_{M}(n) \leq n^{k}+k$ [2]. In other words, this means the language $L(M)$ can be accepted by the Turing machine $M$ in polynomial time. Therefore, $P$ is the complexity class of languages that can be accepted in polynomial time by deterministic Turing machines [7]. A verifier for a language $L$ is a deterministic Turing machine $M$, where

$$
L=\{w: M(w, c)=\text { "yes" for some string } c\} .
$$

We measure the time of a verifier only in terms of the length of $w$, so a polynomial time verifier runs in polynomial time in the length of $w$ [2]. A verifier uses additional information, represented by the symbol $c$, to verify that a string $w$ is a member of $L$. This information is called certificate. $N P$ is also the complexity class of languages defined by polynomial time verifiers [18]. If $N P$ is the class of problems that have succinct certificates, then the complexity class $\operatorname{coNP}$ must contain those problems that have succinct disqualifications [18]. That is, a " $n o$ " instance of a problem in $c o N P$ possesses a short proof of its being a " $n o$ " instance [18].

A function $f: \Sigma^{*} \rightarrow \Sigma^{*}$ is a polynomial time computable function if some deterministic Turing machine $M$, on every input $w$, halts in polynomial time with just $f(w)$ on its tape [19]. Let $\{0,1\}^{*}$ be the infinite set of binary strings, we say that a language $L_{1} \subseteq\{0,1\}^{*}$ is polynomial time reducible to a language $L_{2} \subseteq\{0,1\}^{*}$, written $L_{1} \leq_{p} L_{2}$, if there is a polynomial time computable function $f:\{0,1\}^{*} \rightarrow\{0,1\}^{*}$ such that for all $x \in\{0,1\}^{*}$,

$$
x \in L_{1} \text { if and only if } f(x) \in L_{2} .
$$

An important complexity class is $N P$-complete [13]. A language $L \subseteq\{0,1\}^{*}$ is $N P$-complete if 
- $L \in N P$, and

- $L^{\prime} \leq_{p} L$ for every $L^{\prime} \in N P$.

If $L$ is a language such that $L^{\prime} \leq_{p} L$ for some $L^{\prime} \in N P$-complete, then $L$ is $N P$-hard [13]. Moreover, if $L \in N P$, then $L \in N P$-complete [13]. A principal $N P$-complete problem is $H A M-C Y C L E[7]$.

A simple graph is an undirected graph without multiple edges or loops [7]. An instance of the language $H A M-C Y C L E$ is a simple graph $G=(V, E)$ where $V$ is the set of vertices and $E$ is the set of edges, each edge being an unordered pair of vertices [7]. We say $(u, v) \in E$ is an edge in a simple graph $G=(V, E)$ where $u$ and $v$ are vertices. For a simple graph $G=(V, E)$, a simple cycle in $G$ is a sequence of distinct vertices $\left\langle v_{0}, v_{1}, v_{2}, \ldots, v_{k}\right\rangle$ such that $\left(v_{k}, v_{0}\right) \in E$ and $\left(v_{i-1}, v_{i}\right) \in E$ for $i=1,2, \ldots, k[7]$. A Hamiltonian cycle is a simple cycle of the simple graph which contains all the vertices of the graph. A simple graph that contains a hamiltonian cycle is said to be hamiltonian; otherwise, it is nonhamiltonian [7]. The problem $H A M-C Y C L E$ asks whether a simple graph is hamiltonian [7].

\section{Summary}

In computational complexity theory, a sparse language is a formal language (a set of strings) such that the complexity function, counting the number of strings of length $n$ in the language, is bounded by a polynomial function of $n$. The complexity class of all sparse languages is called SPARSE. SPARSE contains TALLY, the class of unary languages, since these have at most one string of any one length.

Fortune showed in 1979 that if any sparse language is coNP-complete, then $P=N P$ (this is Fortune's theorem) [10]. Mahaney used this to show in 1982 that if any sparse language is $N P$-complete, then $P=N P$ [15]. A simpler proof of this based on left-sets was given by Ogihara and Watanabe in 1991 [17]. Mahaney's argument does not actually require the sparse language to be in $N P$, so there is a sparse $N P$-hard set if and only if $P=N P$ [15].

We create a class with the opposite definition, that is a class of languages that are dense instead of sparse. We show there is a sequence of languages that are in $N P$-complete, but their density grows as much as we go forward into the iteration of the sequence. The first element of the sequence is a variation of the $N P$-complete problem known as HAM-CYCLE [18]. The next element in the sequence is constructed from this new version of $H A M-C Y C L E$. Indeed, each language is created from its previous one in the sequence.

Since the density grows according we move forward into the sequence, then there could be a language so much dense such that its complement might be sparse. Fortunately, we see this property from a language created with the elements of these languages on the sequence when the bit-length $n$ of the binary strings tends to infinity. However, this incredible dense language is still $N P$-complete. Thus, the complement of this language remains in coNP-complete, because the complement of every $N P$-complete language is complete for $c o N P$ [18]. As a consequence of Fortune's theorem, we claim that $P$ could be equal to $N P$, since we show the existence of such sparse complete set for $c o N P$.

\section{$4 \quad$ Results}

- Definition 1. A dense language on $m$ is a formal language (a set of binary strings) where there exists a positive integer $n_{0}$ such that the counting of the number of strings of length $n \geq n_{0}$ in the language is greater than or equal to $2^{n-m}$ where $m$ is a real number and $0<m \leq 1$. The complexity class of all dense languages on $m$ is called DENSE $(m)$. 
- Definition 2. A formal language (a set of binary strings) is in DENSE(0) if for every value of $0<m \leq 1$ that we could choose, then the language is always in DENSE $(m)$.

In this work, we are going to represent the simple graphs with an adjacency-matrix [7]. For the adjacency-matrix representation of a simple graph $G=(V, E)$, we assume that the vertices are numbered $1,2, \ldots,|V|$ in some arbitrary manner. The adjacency-matrix representation of a simple graph $G$ consists of a $|V| \times|V|$ matrix $A=\left(a_{i, j}\right)$ such that $a_{i, j}=1$ when $(i, j) \in E$ and $a_{i, j}=0$ otherwise [7]. In this way, every simple graph of $k$ vertices could be represented by a binary string of $k^{2}$ bits.

Observe the symmetry along the main diagonal of the adjacency matrix in this kind of graph that is called simple. We define the transpose of a matrix $A=\left(a_{i, j}\right)$ to be the matrix $A^{T}=\left(a_{i, j}^{T}\right)$ given by $a_{i, j}^{T}=a_{j, i}$. Hence the adjacency matrix $A$ of a simple graph is its own transpose $A=A^{T}$.

- Definition 3. The language NON-SIMPLE contains all the graph that are represented by an adjacency-matrix $A$ such that $A \neq A^{T}$ or there is some $a_{i, j}=1$ where $i=j$.

- Lemma 4. NON-SIMPLE $\in P$.

Proof. Given a binary string $x$, we can check whether $x$ is an adjacency-matrix which is not equal to its own transpose in time $O\left(|x|^{2}\right)$ just iterating each bit $a_{i, j}$ in $x$ and checking whether $a_{i, j} \neq a_{j, i}$ or $a_{i, j}=1$ when $i=j$ where $|\ldots|$ represents the bit-length function $[7]$.

- Definition 5. The language HAM-CYCLE' contains all the binary strings z such that $z=x y$, the bit-length of $x$ is equal to $(\lfloor\sqrt{|z|}\rfloor)^{2}$ and $x \in H A M-C Y C L E$ or $x \in$ NON-SIMPLE where $y$ could be the empty string when $|\ldots|$ and $\lfloor\ldots\rfloor$ represent the bit-length function and the floor function respectively.

- Lemma 6. $H A M-C Y C L E^{\prime} \in N P-$ complete.

Proof. Given a binary string $z$ such that $z=x y$ and the bit-length of $x$ is equal to $(\lfloor\sqrt{|z|}\rfloor)^{2}$, we can decide in polynomial time whether $x \notin N O N-S I M P L E$ just verifying when $x=x^{T}$ and $a_{i, i}=0$ for all vertex $i$. In this way, we can reduce in polynomial time a simple graph $G=(V, E)$ of $k$ vertices encoded as the binary string $x$ such that when $x$ has $k^{2}$ bits and $x \notin N O N-S I M P L E$ then

$x \in H A M-C Y C L E$ if and only if $x y \in H A M-C Y C L E$ '

where $y$ could be the empty string. In this way, we can reduce in polynomial time each element of $H A M-C Y C L E$ to some element of HAM-CYCLE'. Therefore, HAM-CYCLE' is in NP-hard. Moreover, we can check in polynomial time over a binary string $z$ such that $z=x y$ and the bit-length of $x$ is equal to $(\lfloor\sqrt{|z|}\rfloor)^{2}$ whether $x \in H A M-C Y C L E$ or $x \in$ NON-SIMPLE since HAM-CYCLE $\in N P$ and NON-SIMPLE $\in N P$ because of $P \subseteq N P$ [18]. Consequently, $H A M-C Y C L E$ ' is in $N P$. Hence, $H A M-C Y C L E ' \in N P$-complete.

- Lemma 7. HAM-CYCLE' $\in$ DENSE(1). This would mean the existence of a sufficiently large positive integer $n_{0}^{\prime}$ such that all the binary strings of length $n \geq n_{0}^{\prime}$ which belong to $H A M-C Y C L E$ ' are more than or equal to $2^{n-1}$ elements.

Proof. OEIS A000088 gives some number of graphs on $n$ unlabeled points [21]. For 8 points there are 12346 so just over half the graphs on 8 points are Hamiltonian [21]. For 12 points, there are 152522187830 Hamiltonian graphs out of 165091172592 which would claim that 
over $92 \%$ of the 12 point graphs are Hamiltonian [21]. For $n=2$ there are two graphs, neither of which is Hamiltonian [21]. For $n<8$ over half the graphs are not Hamiltonian [21]. It does not seem surprising that once $n$ gets large most graphs are Hamiltonian [21].

Choosing a graph on $n$ vertices at random is the same as including each edge in the graph with probability $\frac{1}{2}$, independently of the other edges [4]. You get a more general model of random graphs if you choose each edge with probability $p$ [4]. This model is known as $G_{n, p}$ [4]. It turns out that for any constant $p>0$, the probability that $G_{n, p}$ contains a Hamiltonian cycle tends to 1 when $n$ tends to infinity [4]. In fact, this is true whenever $p>\frac{c \times \log n}{n}$ for some constant $c$. In particular this is true for $p=\frac{1}{2}$, which is our case [4].

For all the binary strings $z$ such that $z=x y$ and the bit-length of $x$ is equal to $(\lfloor\sqrt{|z|}\rfloor)^{2}$, the amount of elements of size $|z|$ in $H A M-C Y C L E$ ' is equal to the number of binary strings $x \in H A M-C Y C L E$ or $x \in N O N-S I M P L E$ of size $(\lfloor\sqrt{|z|}\rfloor)^{2}$ multiplied by $2^{|z|-(\lfloor\sqrt{|z|}\rfloor)^{2}}$. Since the number of Hamiltonian graphs increases as much as we go further on $n$, it does not seem surprising either that once $n$ gets large most binary strings belong to HAM-CYCLE' Moreover, the amount of binary strings which have some bit-length $k^{2}$ and belongs to $N O N-S I M P L E$ is considerably superior to the amount of strings with the same bit-length which are valid simple graphs. Actually, we can affirm for a sufficiently large positive integer $n_{0}^{\prime}$, all the binary strings of length $n \geq n_{0}^{\prime}$ which belong to $H A M-C Y C L E$ ' are indeed more than or equal to $2^{n-1}$ elements. In this way, we show that $H A M-C Y C L E^{\prime} \in D E N S E(1)$.

- Definition 8. We will define a sequence of languages $H A M-C Y C L E^{\prime}{ }_{k}$ for every possible integer $1 \leq k$. We state HAM-CYCLE' ${ }_{1}$ as the language HAM-CYCLE'. Recursively, from a language $H A M-C Y C L E{ }^{\prime}$, we define $H A M-C Y C L E^{\prime}{ }_{k+1}$ as follows: A binary string $x y$ complies with $x y \in H A M-C Y C L E^{\prime}{ }_{k+1}$ if and only if $x$ and $y$ are binary strings, $x \in$ $H A M-C Y C L E^{\prime}{ }_{k}$ or $y \in H A M-C Y C L E^{\prime}{ }_{k}$ such that $|x|=\left\lfloor\frac{|x y|}{2}\right\rfloor$ where $|\ldots|$ represents the bit-length function and $\lfloor\ldots\rfloor$ is the floor function.

- Lemma 9. For every integer $1 \leq k, H A M-C Y C L E{ }_{k} \in N P$.

Proof. This is true for $k=1$ as we see in Lemma 6. Every string $x y$ which belongs to $H A M-C Y C L E{ }_{2}$ complies with $x \in H A M-C Y C L E_{1}{ }_{1}$ or $y \in H A M-C Y C L E_{1}{ }_{1}$ such that $|x|=$ $\left\lfloor\frac{\lfloor x y \mid}{2}\right\rfloor$. Moreover, every string $x y$ which belongs to the language $H A M-C Y C L E_{3}{ }_{3}$ complies with $x \in H A M-C Y C L E{ }_{2}$ or $y \in H A M-C Y C L E{ }_{2}$, such that $|x|=\left\lfloor\frac{|x y|}{2}\right\rfloor$. Furthermore, we can extend this property for every positive integer $k>3$ in $H A M-C Y C L E{ }^{\prime}$. Indeed, $H A M-C Y C L E^{\prime}{ }_{k}$ is in $N P$ for every integer $1 \leq k$, since the verification of whether the two substrings are indeed elements of $H A M-C Y C L E^{\prime}{ }_{k-1}$ can be done in polynomial time with the appropriated certificates using the mathematical induction on $k$.

- Theorem 10. For every integer $1 \leq k, H A M-C Y C L E^{\prime}{ }_{k} \in N P$-complete.

Proof. This is true for $k=1$ by the Lemma 6 . Let's assume it is valid for some positive integer $1 \leq k^{\prime}$. Let's prove this for $k^{\prime}+1$. We already know the adjacency-matrix of $n^{2}$ zeros represents a simple graph of $n$ vertices which does not contain any edge. This kind of a simple graph does not belong to $H A M-C Y C L E_{1}{ }_{1}$. As a consequence, this string will not belong to any $H A M-C Y C L E^{\prime}{ }^{\prime}$, because its substrings of a quadratic length are also adjacency-matrix of only zeros. Suppose, we have an instance $y$ of $H A M-C Y C L E{ }^{\prime}{ }^{\prime}$. We can reduce $y$ in $H A M-C Y C L E^{\prime}{ }^{\prime}$ to $z y$ in $H A M-C Y C L E^{\prime}{ }_{k^{\prime}+1}$ such that

$$
y \in H A M-C Y C L E^{\prime}{ }_{k^{\prime}} \text { if and only if } z y \in H A M-C Y C L E^{\prime}{ }^{\prime}+1
$$

where the binary string $z$ is exactly a sequence of $\left\lfloor\frac{|z y|}{2}\right\rfloor$ zeros. We can do this since we already know $z \notin H A M-C Y C L E{ }^{\prime}{ }_{k^{\prime}}$. Certainly, if the membership $z y \in H A M-C Y C L E^{\prime}{ }^{\prime}+1$ is 
true, $z \notin H A M-C Y C L E^{\prime}{ }_{k^{\prime}}$ and $|z|=\left\lfloor\frac{|z y|}{2}\right\rfloor$, then $y \in H A M-C Y C L E^{\prime}{ }_{k^{\prime}}$ also holds according to the Definition 8. Since this reduction remains in polynomial time for every positive integer $1 \leq k^{\prime}$, then we show that $H A M-C Y C L E^{\prime}{ }_{k^{\prime}+1}$ is in $N P$-hard. Moreover, $H A M-C Y C L E^{\prime}{ }_{k^{\prime}+1}$ is also in $N P$-complete, because of the Lemma 9.

- Theorem 11. For every integer $1 \leq k$, if the language $H A M-C Y C L E{ }^{\prime}{ }_{k}$ is in DENSE $\left(k^{\prime}\right)$ for every instance of bit-length $n^{\prime} \geq n_{0}$, then $H A M-C Y C L E^{\prime}{ }_{k+1}$ is in DENSE $\left(\frac{k^{\prime}}{2}\right)$ for every instance of bit-length $n^{\prime} \geq 2 \times n_{0}$.

Proof. If the language $H A M-C Y C L E{ }^{\prime}{ }_{k}$ is in $D E N S E\left(k^{\prime}\right)$ for every instance of bit-length $n^{\prime} \geq n_{0}$, then for every integer $n \geq n_{0}$ the amount of elements of size $n+i$ in $H A M-C Y C L E{ }^{\prime}{ }_{k+1}$ (where $i \geq n_{0}$ and $i=\left\lfloor\frac{n+i}{2}\right\rfloor$ ) is greater than or equal to

$$
2^{i-k^{\prime}} \times 2^{n}+2^{n-k^{\prime}} \times\left(2^{i}-2^{i-k^{\prime}}\right) .
$$

This is because there must be more than or equal to $2^{i-k^{\prime}}$ elements of size $i$ in $H A M-C Y C L E{ }^{\prime} k$ which are prefixes of the binary strings of size $n+i$ in the language $H A M-C Y C L E{ }^{\prime}{ }_{k+1}$. We multiply that amount by $2^{n}$ since this is the number of different combinations of suffixes with length $n$ in the binary strings of size $n+i$. Moreover, there must be more than or equal to $2^{n-k^{\prime}}$ elements of size $n$ in $H A M-C Y C L E{ }^{\prime}{ }_{k}$ which are suffixes of the binary strings of size $n+i$ in $H A M-C Y C L E^{\prime}{ }_{k+1}$. We multiply that amount by $\left(2^{i}-2^{i-k^{\prime}}\right)$ since this is the number of different combinations of prefixes with length $i$ in the binary strings of size $n+i$ just avoiding to count the previous prefixes twice. If we join both properties, we obtain the sum described by the formula above.

Indeed, this formula can be simplified to

$2^{n+i-k^{\prime}}+2^{n+i-k^{\prime}} \times\left(2^{0}-2^{-k^{\prime}}\right)$

and extracting a common factor we obtain

$$
2^{n+i-k^{\prime}} \times\left(1+\left(1-2^{-k^{\prime}}\right)\right)
$$

which is equal to

$$
2^{n+i-k^{\prime}} \times\left(2-\frac{1}{2^{k^{\prime}}}\right)
$$

Nevertheless, for every real number $0<k^{\prime} \leq 1$ we have that

$$
\left(2-\frac{1}{2^{k^{\prime}}}\right) \geq 2^{\frac{k^{\prime}}{2}} .
$$

Certainly, if we multiply both member of the inequality by $2^{k^{\prime}}$, we obtain

$$
\left(2^{k^{\prime}+1}-1\right) \geq 2^{k^{\prime}+\frac{k^{\prime}}{2}}
$$

which is equivalent to

$$
2^{k^{\prime}} \times\left(2-2^{\frac{k^{\prime}}{2}}\right) \geq 1
$$

that it is true for every real number $0<k^{\prime} \leq 1$. We can check in the Figure 1 that the function $f(x)=2^{x} \times\left(2-2^{\frac{x}{2}}\right)$ is greater than or equal to 1 over the interval [0,1]. Thus

$$
2^{n+i-k^{\prime}} \times\left(2-\frac{1}{2^{k^{\prime}}}\right) \geq 2^{n+i-k^{\prime}} \times 2^{\frac{k^{\prime}}{2}}
$$




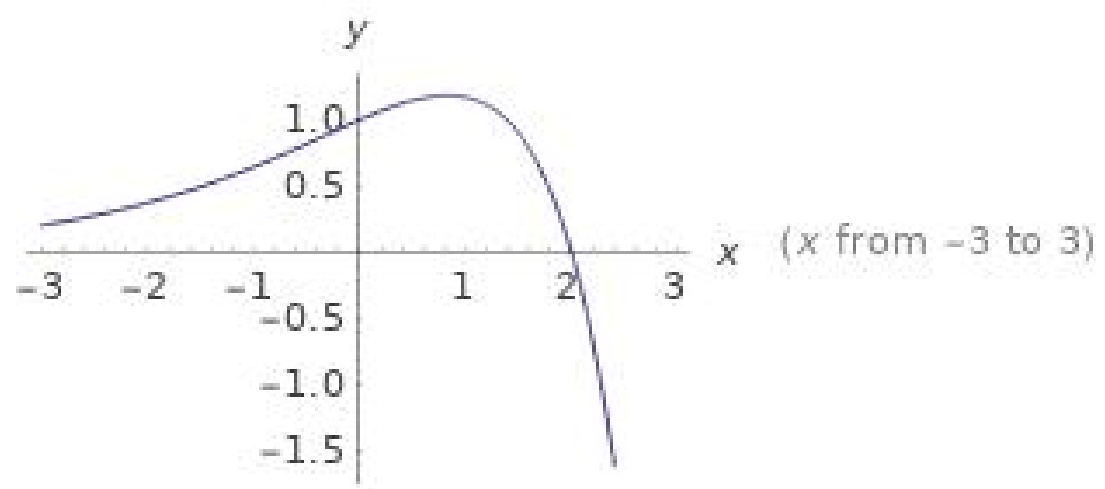

Figure 1 Plot the function $\mathrm{f}(\mathrm{x})$ on the interval $[-3,3]$

where

$$
2^{n+i-k^{\prime}} \times 2^{\frac{k^{\prime}}{2}}=2^{n+i-\left(k^{\prime}-\frac{k^{\prime}}{2}\right)}=2^{n+i-\frac{k^{\prime}}{2}} .
$$

Since there are more than or equal to $2^{n^{\prime}-\left(\frac{k^{\prime}}{2}\right)}$ elements of the language $H A M-C Y C L E{ }^{\prime}{ }_{k+1}$ with length $n^{\prime} \geq 2 \times n_{0}$ therefore, we show that $H A M-C Y C L E^{\prime}{ }_{k+1}$ is in DENSE( $\left.\frac{k^{\prime}}{2}\right)$ for every instance of bit-length $n^{\prime} \geq 2 \times n_{0}$.

- Lemma 12. HAM-CYCLE' ${ }_{k} \in D E N S E\left(\frac{1}{2^{k-1}}\right)$ for every instance of bit-length $n \geq$ $2^{k-1} \times n_{0}^{\prime}$, where the constant $n_{0}^{\prime}$ is the positive integer used in the Definition 1 and Lemma 7 for $H A M-C Y C L E$ '.

Proof. According to the Lemma 7, HAM-CYCLE' ${ }_{1}$ is in $D E N S E(1)$ for every instance of bit-length $n \geq 2^{0} \times n_{0}^{\prime}=n_{0}^{\prime}$. Consequently, due to Theorem 11, HAM-CYCLE' ${ }_{2}$ is in $\operatorname{DENSE}\left(\frac{1}{2}\right)$ for every instance of bit-length $n \geq 2^{1} \times n_{0}^{\prime}$. Moreover, $H A M-C Y C L E_{3}^{\prime}$ is in $\operatorname{DENSE}\left(\frac{1}{4}\right)$ for every instance of bit-length $n \geq 2^{2} \times n_{0}^{\prime}$ and so forth ... and thus, for every language $H A M-C Y C L E^{\prime}{ }_{k}$, we have that $H A M-C Y C L E^{\prime}{ }_{k} \in D E N S E\left(\frac{1}{2^{k-1}}\right)$ for every instance of bit-length $n \geq 2^{k-1} \times n_{0}^{\prime}$.

- Definition 13. We will define a language $H A M-C Y C L E_{\infty}{ }_{\infty}$ as follows: $A$ binary string $x$ complies with $x \in H A M-C Y C L E^{\prime}{ }_{\infty}$ if and only if we obtain that $x \in H A M-C Y C L E^{\prime}{ }_{k}$ and $2^{k-1} \times n_{0}^{\prime} \leq|x|<2^{k} \times n_{0}^{\prime}$ where $|\ldots|$ represents the bit-length function and the constant $n_{0}^{\prime}$ is the positive integer used in the Definition 1 and Lemma 7 for HAM-CYCLE'.

- Lemma 14. $H A M-C Y C L E_{\infty}^{\prime} \in N P$.

Proof. We can calculate the value of $k$ from some binary string $x$ that is approximately $\left\lceil\log _{2}\left(\frac{|x|}{n_{0}^{\prime}}\right)\right\rceil$, where $\lceil\ldots\rceil$ is the ceiling function. In this way, we should know if $x \in H A M-C Y C L E^{\prime}{ }_{\infty}$, then $x \in H A M-C Y C L E^{\prime}{ }_{k}$. However, for every positive integer $k$, we can check in polynomial time whether $x \in H A M-C Y C L E^{\prime}{ }_{k}$ just splitting the binary string $x$ into the following substrings $x=x_{1} x_{2} x_{3} \ldots x_{2^{k-1}}$ and verifying later whether $x_{1} \in H A M-C Y C L E^{\prime}{ }_{1}$ or $x_{2} \in H A M-C Y C L E_{1}{ }_{1}$ or $x_{3} \in H A M-C Y C L E^{\prime}{ }_{1}$ and so forth ... until we finally check whether $x_{2^{k-1}} \in H A M-C Y C L E_{1}{ }_{1}$ where $2^{k-1}$ is polynomially bounded by the bit-length string $|x|$. Indeed, the language $H A M-C Y C L E^{\prime}{ }_{\infty}$ is in $N P$, because the verification of whether the whole string or a polynomially amount of substrings are indeed elements of $H A M-C Y C L E_{1}$, can be done in polynomial time with the appropriated certificates. 
- Theorem 15. HAM-CYCLE' ${ }_{\infty} \in N P-$ complete.

Proof. We already know the adjacency-matrix of $n^{2}$ zeros represents a simple graph of $n$ vertices which does not contain any edge. This kind of a simple graph does not belong to $H A M-C Y C L E{ }_{1}$. Suppose, we have an instance $y$ of $H A M-C Y C L E_{1}$. We can reduce $y$ in $H A M-C Y C L E{ }_{1}^{\prime}$ to $z y$ in $H A M-C Y C L E_{\infty}^{\prime}$ such that

$y \in H A M-C Y C L E{ }_{1}$ if and only if $z y \in H A M-C Y C L E_{\infty}{ }_{\infty}$

where $z$ is a binary string of a sequence of zeros such that $2^{k-1} \times n_{0}^{\prime} \leq|z y|<2^{k} \times n_{0}^{\prime}$ and the membership in $z y \in H A M-C Y C L E^{\prime}{ }_{k}$ implies that $y \in H A M-C Y C L E{ }_{1}$, where the constant $n_{0}^{\prime}$ is the positive integer used in the Definition 1 and Lemma 7 for HAM-CYCLE'. We claim that the bit-length of $z y$ is polynomially bounded by $|y|$. Certainly, the bit-length of $z$ is bounded by $2^{k-1} \times n_{0}^{\prime}$ which is polynomially bounded by $|y|$ since $k \approx\left\lceil\log _{2}\left(\frac{|z y|}{n_{0}^{\prime}}\right)\right\rceil$, where $\lceil\ldots\rceil$ is the ceiling function. That expression is equivalent to $2^{k} \approx \frac{|y|+2^{k-1} \times n_{0}^{\prime}}{n_{0}^{\prime}}$ which means that $\frac{|y|}{2^{k} \times n_{0}^{\prime}} \approx 1$ and thus, our claim should be true. In this way, we show that $H A M-C Y C L E_{\infty}^{\prime}$ is in NP-hard. Moreover, we demonstrate $H A M-C Y C L E_{\infty}^{\prime}$ is also in $N P$-complete, because of the Lemma 14.

- Lemma 16. HAM-CYCLE ${ }_{\infty}^{\prime} \in \operatorname{DENSE}(0)$ since $H A M-C Y C L E_{\infty}^{\prime} \in \operatorname{DENSE}\left(\frac{2 \times n_{0}^{\prime}}{n}\right)$, where $n$ is the bit-length of the binary strings and the constant $n_{0}^{\prime}$ is the positive integer used in the Definition 1 and Lemma 7 for HAM-CYCLE'.

Proof. When $k$ tends to infinity, then $\frac{1}{2^{k-1}}$ tends to 0 . In this way, we obtain that $H A M-C Y C L E^{\prime}{ }_{k} \in D E N S E(0)$ as a consequence of the Lemma 12. HAM-CYCLE' contains the elements of the languages $H A M-C Y C L E{ }^{\prime}$ into the interval of the binary strings between the bit-length $2^{k-1} \times n_{0}^{\prime} \leq n<2^{k} \times n_{0}^{\prime}$. Those elements will have a bit-length greater than $2^{k-1} \times n_{0}^{\prime}$ and by the Lemma 12 the density in the interval will be $\operatorname{DENSE}\left(\frac{1}{2^{k-1}}\right)$ which is equivalent to

$$
\operatorname{DENSE}\left(\frac{1}{2^{\log _{2}\left(\frac{n}{n_{0}^{\prime}}\right)-1}}\right)
$$

in the same interval of the binary strings between the bit-length $2^{k-1} \times n_{0}^{\prime} \leq n<2^{k} \times n_{0}^{\prime}$. In this way, we know that

$$
\operatorname{DENSE}\left(\frac{1}{2^{\log _{2}\left(\frac{n}{n_{0}^{\prime}}\right)-1}}\right)=\operatorname{DENSE}\left(\frac{2}{2^{\log _{2}\left(\frac{n}{n_{0}^{\prime}}\right)}}\right)=\operatorname{DENSE}\left(\frac{2 \times n_{0}^{\prime}}{n}\right) .
$$

- Theorem 17. There is a sparse language in coNP-complete.

Proof. In the Lemma 16, the complement of $H A M-C Y C L E_{\infty}{ }_{\infty}$ could be sparse. Thus, the complexity of counting the number of strings with length $n$ in the complement of this language could be bounded by a polynomial function of $n$. Actually, when a language is sparse, then its complement is in $D E N S E(0)$ [15]. Indeed, the sparse languages are called sparse because there are a total of $2^{n}$ strings of length $n$, and if a language only contains polynomially many of these, then the proportion of strings of length $n$ that it contains rapidly goes to zero as $n$ grows (which means its complement should be in $D E N S E(0)$ ) [15]. Furthermore, we claim that if a language belongs to $\operatorname{DENSE}\left(\frac{\alpha}{n}\right)$, then its complement should be sparse, where 
$\alpha=2 \times n_{0}^{\prime}$ is a constant and $n$ is the bit-length of the binary strings. Certainly, we know by the L'Hospital's rule that

$$
\lim _{n \rightarrow \infty} \frac{n^{c}}{2^{n}}=0
$$

for some positive constant $c$ [20]. The number of binary strings of bit-length $n$ in the complement of $H A M-C Y C L E$ ' ${ }_{\infty}$ would be lesser than or equal to $2^{n}-2^{n-\frac{\alpha}{n}}$ for a sufficiently large value of $n$. However, it is enough to show that

$$
\lim _{n \rightarrow \infty} \frac{2^{n}-2^{n-\frac{\alpha}{n}}}{2^{n}}=\lim _{n \rightarrow \infty}\left(1-\frac{1}{2^{\frac{\alpha}{n}}}\right)=0 .
$$

We claim that the expressions $\left(1-\frac{1}{2^{\frac{\alpha}{n}}}\right)$ and $\frac{n^{c}}{2^{n}}$ rapidly go to zero as $n$ grows in a very simultaneous way. In addition, according to Theorem 15, the complement of this language $H A M-C Y C L E{ }_{\infty}$ must be in coNP-complete, because the complements of the NP-complete problems are complete for $\operatorname{coNP}[18]$.

- Lemma 18. $P=N P$.

Proof. By the Fortune's theorem, if any sparse language is coNP-complete, then $P=N P$ [10]. As result of Theorem 17, there exists a possible sparse language in coNP-complete. Finally, we claim that $P$ could be equal to $N P$.

\section{Discussion}

A logarithmic space Turing machine has a read-only input tape, a write-only output tape, and a read/write work tape [19]. The work tape may contain $O(\log n)$ symbols [19]. In computational complexity theory, LOGSPACE is the complexity class containing those decision problems that can be decided by a logarithmic space Turing machine which is deterministic [18]. Whether LOGSPACE $=P$ is another fundamental question that it is as important as it is unresolved [18].

In 1999, Jin-Yi Cai and D. Sivakumar, building on work by Ogihara, showed that if there exists a sparse $P$-complete problem, then LOGSPACE $=P[5]$. We might extend the proof of this paper to show the same result on $P$ and claim that LOGSPACE $=P$. Certainly, we might only need to find some P-complete which belongs to DENSE(1) because the $P$-completeness is closed under complement [18]. Indeed, the other steps of that possible proof might be similar to the arguments that we follow in this paper. Consequently, this work might help us not only to solve $P$ versus $N P$, but also LOGSPACE versus $P$.

\section{Conclusions}

No one has been able to find a polynomial time algorithm for any of more than 300 important known $N P$-complete problems [11]. A proof of $P=N P$ will have stunning practical consequences, because it leads to efficient methods for solving some of the important problems in NP [6]. The consequences, both positive and negative, arise since various $N P$-complete problems are fundamental in many fields [6]. This result explicitly concludes with the evidence of a feasible solution for the $N P$-complete problems.

Cryptography, for example, relies on certain problems being difficult. A constructive and efficient solution to an $N P$-complete problem such as $3 S A T$ will break most existing cryptosystems including: Public-key cryptography [14], symmetric ciphers [16] and one-way 
functions used in cryptographic hashing [8]. These would need to be modified or replaced by information-theoretically secure solutions not inherently based on $P-N P$ equivalence.

Learning becomes easy by using the principle of Occam's razor-we simply find the smallest program consistent with the data [9]. Near perfect vision recognition, language comprehension and translation and all other learning tasks become trivial [9]. We would also have much better predictions of weather and earthquakes and other natural phenomenon [9].

There are enormous positive consequences that will follow from rendering tractable many currently mathematically intractable problems. For instance, many problems in operations research are $\mathrm{NP}$-complete, such as some types of integer programming and the traveling salesman problem [11]. Efficient solutions to these problems have enormous implications for logistics [6]. Many other important problems, such as some problems in protein structure prediction, are also $N P$-complete, so this will spur considerable advances in biology [3].

But such changes may pale in significance compared to the revolution an efficient method for solving $\mathrm{NP}$-complete problems will cause in mathematics itself. Research mathematicians spend their careers trying to prove theorems, and some proofs have taken decades or even centuries to find after problems have been stated. For instance, Fermat's Last Theorem took over three centuries to prove. A method that is guaranteed to find proofs to theorems, should one exist of a "reasonable" size, would essentially end this struggle.

\section{References}

1 Scott Aaronson. P $\stackrel{?}{=}$ NP. Electronic Colloquium on Computational Complexity, Report No. 4, 2017.

2 Sanjeev Arora and Boaz Barak. Computational complexity: a modern approach. Cambridge University Press, 2009.

3 Bonnie Berger and Tom Leighton. Protein folding in the hydrophobic-hydrophilic (HP) model is NP-complete. Journal of Computational Biology, 5(1):27-40, 1998. doi:10.1145/279069. 279080.

4 Béla Bollobás. Random Graphs. Cambridge Studies in Advanced Mathematics. Cambridge University Press, 2 edition, 2001. doi:10.1017/CB09780511814068.

5 Jin-Yi Cai and D. Sivakumar. Sparse hard sets for P: resolution of a conjecture of Hartmanis. Journal of Computer and System Sciences, 58(2):280-296, 1999. doi:10.1006/jcss.1998. 1615.

6 Stephen A Cook. The P versus NP Problem, April 2000. at http://www.claymath.org/ sites/default/files/pvsnp.pdf.

7 Thomas H Cormen, Charles E Leiserson, Ronald L Rivest, and Clifford Stein. Introduction to Algorithms. The MIT Press, 3rd edition, 2009.

8 Debapratim De, Abishek Kumarasubramanian, and Ramarathnam Venkatesan. Inversion attacks on secure hash functions using SAT solvers. In International Conference on Theory and Applications of Satisfiability Testing, pages 377-382. Springer, 2007.

9 Lance Fortnow. The Status of the P Versus NP Problem. Commun. ACM, 52(9):78-86, September 2009. doi:10.1145/1562164.1562186.

10 S. Fortune. A note on sparse complete sets. SIAM Journal on Computing, 8(3):431-433, 1979. doi:10.1137/0208034.

11 Michael R Garey and David S Johnson. Computers and Intractability: A Guide to the Theory of NP-Completeness. San Francisco: W. H. Freeman and Company, 1 edition, 1979.

12 William I Gasarch. Guest column: The second $\mathrm{P} \stackrel{?}{=}$ NP poll. ACM SIGACT News, 43(2):53-77, 2012.

13 Oded Goldreich. P, NP, and NP-Completeness: The basics of computational complexity. Cambridge University Press, 2010. 
14 Satoshi Horie and Osamu Watanabe. Hard instance generation for SAT. Algorithms and Computation, pages 22-31, 1997. doi:10.1007/3-540-63890-3\_4.

15 S. R. Mahaney. Sparse complete sets for NP: Solution of a conjecture by Berman and Hartmanis. Journal of Computer and System Sciences, 25:130-143, 1982. doi:10.1016/0022-0000(82) 90002-2.

16 Fabio Massacci and Laura Marraro. Logical cryptanalysis as a SAT problem. Journal of Automated Reasoning, 24(1):165-203, 2000. doi:10.1023/A:1006326723002.

17 M. Ogiwara and O. Watanabe. On polynomial time bounded truth-table reducibility of NP sets to sparse sets. SIAM Journal on Computing, 20:471-483, 1991. doi:10.1137/0220030.

18 Christos H Papadimitriou. Computational complexity. Addison-Wesley, 1994.

19 Michael Sipser. Introduction to the Theory of Computation, volume 2. Thomson Course Technology Boston, 2006.

20 Angus E Taylor. L'Hospital's Rule. The American Mathematical Monthly, 59(1):20-24, 1952. doi:10.1080/00029890.1952.11988058.

21 The On-Line Encyclopedia of Integer Sequences. Number of graphs on n unlabeled nodes, August 2018. at http://oeis.org/A000088. 